\title{
Editorial: 20th anniversary of Finance and Stochastics
}

More than a century ago, Louis Bachelier published his thesis "Théorie de la spéculation”, Ann. Sci. École Norm. Sup. 3 (1900), in which he invented Brownian motion as a tool for the analysis of financial markets. A.N. Kolmogorov, in his own landmark work "Über die analytischen Methoden in der Wahrscheinlichkeitsrechnung", Math. Annalen 104 (1931), pp. 415-458, credits Bachelier with the first systematic study of stochastic processes in continuous time. But in addition, Bachelier's thesis marks the beginning of the theory of option pricing, now an integral part of modern finance. Thus, the year 1900 may be considered as the birth date of both Finance and Stochastics.

It took almost a century after Bachelier's pioneering work to the birth of Finance and Stochastics as a scientific journal. The initiative to found a high quality journal with special emphasis on the interface between Finance and Stochastics was started in the spring of 1995 by Dieter Sondermann, with strong support by many other members of the future editorial board and by Werner A. Müller and Catriona Byrne from the publisher Springer. From the start, this project could rely on the mathematical expertise of a large group of leading experts in Stochastics with an active research interest in Finance. The start of Finance and Stochastics in 1996 coincided with the foundation of the Bachelier Finance Society. Both the Journal and the Society serve the same aim: to promote the fruitful interaction of the two disciplines Finance and Stochastics.

The purpose of Finance and Stochastics is to provide a high standard publication forum for research in all areas of finance based on stochastic methods and on specific topics in mathematics (in particular probability theory, statistics and stochastic analysis) motivated by the analysis of problems in finance. Finance and Stochastics encompasses - but is not limited to - the following fields:

- theory and analysis of financial markets

- continuous time finance 
- derivatives research

- insurance in relation to finance

- portfolio selection

- credit and market risks

- term structure models

- statistical and empirical financial studies based on advanced stochastic methods

- numerical and stochastic solution techniques for problems in finance

- intertemporal economics, uncertainty and information in relation to finance

In summary, Finance and Stochastics serves as a publication platform for both theoretical and applied financial economists using advanced stochastic methods and researchers in stochastics motivated by and interested in applications in finance and insurance.

This year, Finance and Stochastics is celebrating its 20th anniversary. The current issue is the last of Volume 20, and it is time for a retrospective look at the developments and achievements in the last 20 years. The research area of mathematical finance has evolved tremendously. It has celebrated some enormous successes; it has suffered from many criticisms during times of financial distress; and it is still very active in formulating and answering new questions and challenges from both applications and theory. Finance and Stochastics has established itself as one of the leading journals in this active field, and its success can be traced back to many contributing sources. We are grateful to the members of the Editorial Board and in particular the Co-Editors, the referees for their reviewing work, the publisher Springer for its professional support, and the authors for submitting high quality papers, including those whose papers could not be published. We are confident that the success story of $\mathrm{Fi}^{-}$ nance and Stochastics will continue, and we wish the journal all the best for the many years still to come.

Acknowledgements This editorial has benefited from many earlier editorials published in Finance and Stochastics, as can be clearly seen by going back to earlier issues. The project of this journal was thought out very well from its beginning, and we decided that it is sometimes better not to add too much to a success story.

Dieter Sondermann, founding editor

Zürich, October 2016

Martin Schweizer, current editor 